(C) 2012 IEEE. Personal use of this material is permitted. Permission from IEEE must be obtained for all other uses, in any current or future media, including reprinting/republishing this material for advertising or

promotional purposes, creating new collective works, for resale or redistribution to servers or lists, or reuse of any copyrighted component of this work in other works.

\title{
Event-triggered PI control: Saturating actuators and anti-windup compensation
}

\author{
Daniel Lehmann, Georg Alexander Kiener and Karl Henrik Johansson
}

\begin{abstract}
Event-triggered control aims at reducing the communication load over the feedback link in networked control systems by adapting the information exchange to the current needs. This paper investigates the consequences of actuator saturation on the behavior of event-triggered PI control. Stability properties are derived using linear matrix inequalities (LMIs) which show how the stability of the event-triggered control loop depends on the selection of the event threshold. In order to overcome the potential performance degradation due to integrator windup caused by actuator saturation, the proposed scheme is extended by incorporating a static antiwindup mechanism. The results are illustrated by simulations.
\end{abstract}

\section{INTRODUCTION}

\section{A. Event-triggered control}

In the context of networked control systems [13], eventtriggered control has gained attention by considering this control strategy as a means to reduce the communication load of the network ([2], [3], [5], [17]). The main aim to be reached by this feedback structure is the adaptation of the communication among the components of the feedback loop to the current needs. In fact, by reducing the information exchange to the minimum communication that is necessary to ensure the required system performance, an overload of the digital communication network can be avoided.

The event-triggered control loop as considered in this paper is depicted in Fig. 1. It consists of

- the plant with state $\boldsymbol{x}(t)$, exogenous disturbance $\boldsymbol{d}(t)$ and input $\tilde{\boldsymbol{u}}(t)$ subject to actuator limitations,

- an event generator which invokes a communication whenever the state $\boldsymbol{x}(t)$ satisfies a certain event condition,

- and a PI controller which gets the reference input $\boldsymbol{w}(t)$ and continuously produces the control input $\boldsymbol{u}(t)$.

The controller and the sensor node are connected by means of a digital network. Only at event times $t_{k}(k=0,1,2, \ldots)$ determined by the event generator, the measured state $\boldsymbol{x}\left(t_{k}\right)$ is sent from the sensor node towards the controller which is indicated by the dashed lines. The solid lines indicate continuous-time signals.

\section{B. Literature}

Up to now, most of the approaches dealing with eventtriggered control, e.g., [1], [6], [8], [12], [19], consider

This work was supported by the VINNOVA project WiComPI, the Knut and Alice Wallenberg Foundation, the Swedish Research Council, and the HYCON2 EU project.

D. Lehmann, G. A. Kiener, and K. H. Johansson are with the Automatic Control Laboratory, KTH Royal Institute of Technology, Stockholm, 10044 Sweden dlehmann, gakiener, kallej@kth.se

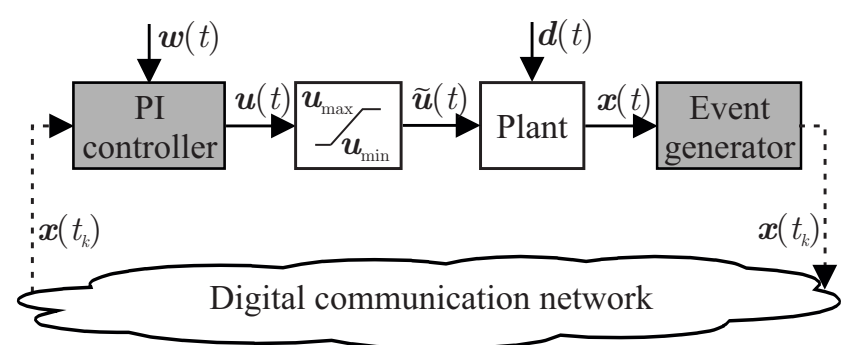

Fig. 1. Event-triggered PI-control loop.

a simple proportional controller. The analysis is primarily concentrated on showing the stability as well as the communication properties of the event-triggered control loop.

However, in order to apply event-triggered control in practice further requirements become important especially setpoint tracking for constant reference and disturbance signals. In this context, [2], [14], [18] showed by simulations that event-triggered PID control is able to significantly reduce the computational and communication effort while only slightly degrading the control performance.

A model-based approach to event-triggered PI control has been presented in [10]. It provides a theoretical framework to analyze the stationary behavior of the event-triggered control loop for constant and time-varying reference and disturbance signals. However, the scheme proposed is computationally demanding as it requires to run the model used for producing the control input $\boldsymbol{u}(t)$ both on the actuator and on the sensor node.

In [7] a very general dynamical event-triggered controller has been considered. By using an extended event-generating mechanism which simultaneously monitors the evolution of the measured output $\boldsymbol{y}(t)$ and the control input $\boldsymbol{u}(t)$, the behavior of the event-triggered control loop was analyzed in terms of its stability and $\mathcal{L}_{\infty}$ properties resulting in LMI conditions.

Two general problems of event-triggered PI control in terms of setpoint tracking are the sticking effect and large stationary oscillations (see [2], [18]). To overcome these problems a modified integrator part of the event-triggered PI controller has been introduced in [16]. It has been shown that for stable first-order systems setpoint tracking can be guaranteed by using the proposed scheme.

Additionally, it is well known from continuous-time control that by using a PI controller the closed-loop behavior might be significantly deteriorated whenever the actuators saturate due to physical or safety constraints (see [4], [15], 
[20]). Even though actuator saturation almost always occurs in practical applications, its effect on event-triggered PI control has only been studied in [9]. There, it has been shown by simulations that actuator saturation has severe consequences with respect to the behavior of the eventtriggered PI-control loop which depend on the selection of the event threshold.

\section{Contributions of this paper}

This paper extends the work in [9] by providing a theoretical framework for dealing with event-triggered PI control subject to actuator saturation. The contributions of this paper are the following:

1) It derives a continuous-time state-space model which shows how event-triggered PI control is affected by actuator saturation.

2) It presents a stability condition based on a sector nonlinearity model approach (proposed in [15] for continuous-time systems) which provides stability regions for the event-triggered PI-control loop by means of LMI conditions (Theorem 1).

3) It is shown that Zeno behavior can be excluded as a lower bound on the minimum inter-event time can be determined (Theorem 2).

4) To overcome a potential performance degradation caused by actuator saturation, it extends the eventtriggered control loop by incorporating a static antiwindup mechanism (Corollaries 1,2).

5) It is shown by simulations how the stability regions are affected by the event threshold and how the antiwindup mechanism improves the results.

The remainder of this paper is organized as follows. Section II introduces some basic notations. The effect of actuator saturation is investigated in Sec. III. The extension by incorporating a static anti-windup mechanism is discussed in Sec. IV. Section V presents a numerical example.

\section{PRELIMINARIES}

Throughout this paper a scalar is denoted by italic letters $(x \in \mathbb{R})$, a vector by bold italic letters $\left(\boldsymbol{x} \in \mathbb{R}^{n}\right)$, a matrix by upper-case bold italic letters $\left(\boldsymbol{A} \in \mathbb{R}^{n \times n}\right)$ and a signal at time $t \in \mathbb{R}_{+}$by $\boldsymbol{x}(t)$, where $\boldsymbol{x}_{0}$ is defined as the initial signal value at time $t=0$. The $i$-th element of a vector $\boldsymbol{x}$ is denoted by $x_{i}$ and the $i$-th row or column of a matrix $\boldsymbol{A}$ by $\boldsymbol{A}_{i}$. The absolute value of a scalar $x$ is denoted by $|x|$ and $\|\boldsymbol{x}\|$ and $\|\boldsymbol{A}\|$ are used to denote the Euclidean vector norm or induced matrix norm. Symmetric matrices of the form $\left(\begin{array}{cc}\boldsymbol{A} & \boldsymbol{B} \\ \boldsymbol{B}^{\mathrm{T}} & \boldsymbol{C}\end{array}\right)$ are written as $\left(\begin{array}{cc}\boldsymbol{A} & \boldsymbol{B} \\ \star & \boldsymbol{C}\end{array}\right)$ with ()$^{\mathrm{T}}$ denoting the transpose of a vector or a matrix. $\boldsymbol{A}>0$ and $\boldsymbol{A} \geq 0$ mean that the matrix $\boldsymbol{A}$ is positive definite or positive semidefinite, respectively. Accordingly, $\boldsymbol{A}<0$ and $\boldsymbol{A} \leq 0$ mean that the matrix $\boldsymbol{A}$ is negative definite or negative semidefinite, respectively.

The plant is assumed to be given by the linear state-space representation

$$
\dot{\boldsymbol{x}}(t)=\boldsymbol{A} \boldsymbol{x}(t)+\boldsymbol{B} \tilde{\boldsymbol{u}}(t)+\boldsymbol{E} \boldsymbol{d}(t), \quad \boldsymbol{x}(0)=\boldsymbol{x}_{0}
$$

where $\boldsymbol{x} \in \mathbb{R}^{n}$ denotes the state of the plant which is assumed to be measurable and $\tilde{\boldsymbol{u}} \in \mathbb{R}^{m}$ is the input of the plant given by $\tilde{\boldsymbol{u}}(t)=\operatorname{sat}(\boldsymbol{u}(t))$ which represents a symmetric saturation nonlinearity according to

$$
\operatorname{sat}\left(u_{i}(t)\right)= \begin{cases}u_{0 i}, & u_{i}(t)>u_{0 i} ; \\ u_{i}(t), & -u_{0 i} \leq u_{i}(t) \leq u_{0 i} ; \\ -u_{0 i}, & u_{i}(t)<-u_{0 i},\end{cases}
$$

for $i=1,2, \ldots, m$. The disturbance $\boldsymbol{d} \in \mathbb{R}^{l}$ is assumed to be an amplitude bounded exogenous signal belonging to the set

$$
\boldsymbol{d} \in \mathcal{D}=\left\{\boldsymbol{d}: \boldsymbol{d}^{\mathrm{T}} \boldsymbol{H} \boldsymbol{d} \leq \beta^{-1}\right\}
$$

with $\boldsymbol{H}=\boldsymbol{H}^{\mathrm{T}}>0$ and $\beta>0$. The pair $(\boldsymbol{A}, \boldsymbol{B})$ is assumed to be controllable.

\section{EVENT-TRIGGERED PI CONTROL SUBJECT TO ACTUATOR SATURATION}

\section{A. Components of the event-triggered PI-control loop}

Event generator: The event generator uses extended deadband sampling (see [14]). A new communication event is invoked whenever the state error $\boldsymbol{e}(t)$ defined as

$$
\boldsymbol{e}(t)=\boldsymbol{x}(t)-\boldsymbol{x}\left(t_{k}\right),
$$

reaches the boundary of the set

$$
\mathcal{E}=\left\{\boldsymbol{e}: \boldsymbol{e}^{\mathrm{T}} \boldsymbol{R} \boldsymbol{e} \leq \delta^{-1}\right\},
$$

i.e., $t:=t_{k+1}$ if

$$
\boldsymbol{e}(t) \in \partial \mathcal{E}=\left\{\boldsymbol{e}: \boldsymbol{e}^{\mathrm{T}} \boldsymbol{R} \boldsymbol{e}=\delta^{-1}\right\}
$$

with $\delta^{-1}>0$ the event threshold and $\boldsymbol{R}=\boldsymbol{R}^{\mathrm{T}}>0$.

PI controller: The controller gets new information about the current plant state $\boldsymbol{x}(t)$ only at event times $t_{k}$ $(k=0,1,2, \ldots)$. It can be described in the time interval $\left[t_{k}, t_{k+1}\right)$ by

$$
\begin{aligned}
\dot{\boldsymbol{x}}_{\mathrm{I}}(t) & =\boldsymbol{x}\left(t_{k}\right)-\boldsymbol{w}(t), \quad \boldsymbol{x}_{\mathrm{I}}\left(t_{k}\right)=\boldsymbol{x}_{\mathrm{I} k} \\
\boldsymbol{u}(t) & =\boldsymbol{K}_{\mathrm{I}} \boldsymbol{x}_{\mathrm{I}}(t)+\boldsymbol{K}_{\mathrm{P}}\left(\boldsymbol{x}\left(t_{k}\right)-\boldsymbol{w}(t)\right), \quad t \in\left[t_{k}, t_{k+1}\right)
\end{aligned}
$$

with $\boldsymbol{x}_{\mathrm{I}} \in \mathbb{R}^{n}$ the controller (integrator) state. By replacing $\boldsymbol{x}\left(t_{k}\right)$ according to (3), the equivalent continuous-time representation

$$
\begin{aligned}
\dot{\boldsymbol{x}}_{\mathrm{I}}(t) & =\boldsymbol{x}(t)-\boldsymbol{e}(t)-\boldsymbol{w}(t), \quad \boldsymbol{x}_{\mathrm{I}}(0)=\boldsymbol{x}_{\mathrm{I} 0} \\
\boldsymbol{u}(t) & =\boldsymbol{K}_{\mathrm{I}} \boldsymbol{x}_{\mathrm{I}}(t)+\boldsymbol{K}_{\mathrm{P}}(\boldsymbol{x}(t)-\boldsymbol{e}(t)-\boldsymbol{w}(t))
\end{aligned}
$$

$(t \geq 0)$ is obtained.

\section{B. Model of the closed-loop system}

With plant (1) and controller (4), (5) and by introducing the augmented state vector

$$
\boldsymbol{x}_{\mathrm{a}}(t)=\left(\begin{array}{c}
\boldsymbol{x}(t) \\
\boldsymbol{x}_{\mathrm{I}}(t)
\end{array}\right)
$$

the event-triggered PI-control loop is described by the statespace model

$$
\begin{aligned}
\dot{\boldsymbol{x}}_{\mathrm{a}}(t)= & \boldsymbol{A}_{\mathrm{a}} \boldsymbol{x}_{\mathrm{a}}(t)+\boldsymbol{B}_{\mathrm{a}} \operatorname{sat}(\boldsymbol{u}(t))+\boldsymbol{D}_{\mathrm{a}} \boldsymbol{w}(t) \\
& +\boldsymbol{F}_{\mathrm{a}} \boldsymbol{e}(t)+\boldsymbol{E}_{\mathrm{a}} \boldsymbol{d}(t), \boldsymbol{x}_{\mathrm{a}}(0)=\boldsymbol{x}_{\mathrm{a} 0} \\
\boldsymbol{u}(t)= & \boldsymbol{K} \boldsymbol{x}_{\mathrm{a}}(t)-\boldsymbol{K}_{\mathrm{P}} \boldsymbol{e}(t)-\boldsymbol{K}_{\mathrm{P}} \boldsymbol{w}(t)
\end{aligned}
$$




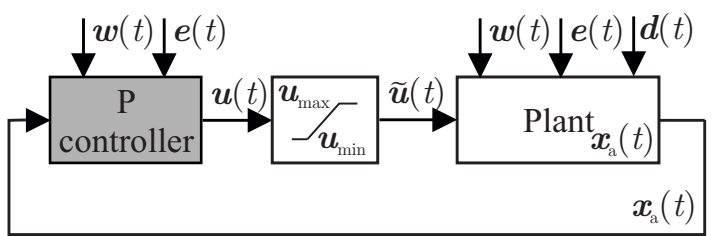

Fig. 2. Continuous-time representation of the event-triggered PI-control loop with augmented plant.

with

$$
\begin{aligned}
& \boldsymbol{A}_{\mathrm{a}}=\left(\begin{array}{cc}
\boldsymbol{A} & \boldsymbol{O} \\
\boldsymbol{I} & \boldsymbol{O}
\end{array}\right), \boldsymbol{B}_{\mathrm{a}}=\left(\begin{array}{c}
\boldsymbol{B} \\
\boldsymbol{O}
\end{array}\right), \boldsymbol{E}_{\mathrm{a}}=\left(\begin{array}{c}
\boldsymbol{E} \\
\boldsymbol{O}
\end{array}\right) \\
& \boldsymbol{D}_{\mathrm{a}}=\boldsymbol{F}_{\mathrm{a}}=\left(\begin{array}{c}
\boldsymbol{O} \\
-\boldsymbol{I}
\end{array}\right), \boldsymbol{K}=\left(\begin{array}{ll}
\boldsymbol{K}_{\mathrm{P}} & \boldsymbol{K}_{\mathrm{I}}
\end{array}\right) .
\end{aligned}
$$

Note that analogously to the controller (4), (5), the statespace model (6), (7) provides a continuous-time representation of the event-triggered PI-control loop (Fig. 1). Here, the augmented plant additionally includes the controller dynamics and both the augmented plant and the controller depend on the state error signal $e(t)$ which is illustrated in Fig. 2. Moreover, the saturation is directly affected by the state error $\boldsymbol{e}(t)$ through the input $\boldsymbol{u}(t)$ according to (7).

\section{Deadzone nonlinearity}

By transforming the saturation nonlinearity $\operatorname{sat}(\boldsymbol{u})$ according to

$$
\phi(\boldsymbol{u})=\operatorname{sat}(\boldsymbol{u})-\boldsymbol{u}
$$

the deadzone nonlinearity $\phi(\boldsymbol{u})$ is introduced. The main benefit of this transformation is that it allows to use a modified sector condition. This condition specifically applies to deadzone nonlinearities potentially resulting in less conservative results compared to using classical sector conditions [15].

Lemma 1: [15] If $\boldsymbol{v} \in \mathbb{R}^{m}$ and $\boldsymbol{z} \in \mathbb{R}^{m}$ are elements of the set

$$
\mathcal{R}_{\mathcal{S}}(\|\boldsymbol{v}-\boldsymbol{z}\|)=\left\{\boldsymbol{v}, \boldsymbol{z}:\left|v_{i}-z_{i}\right| \leq u_{0 i}, \quad \forall i=1,2, \ldots m\right\},
$$

then the nonlinearity $\phi(\boldsymbol{u})$ satisfies the inequality

$$
\phi(\boldsymbol{v})^{\mathrm{T}} \boldsymbol{T}(\phi(\boldsymbol{v})+\boldsymbol{z}) \leq 0
$$

for any positive definite diagonal matrix $\boldsymbol{T} \in \mathbb{R}^{m \times m}$. Replacing the saturation nonlinearity $\operatorname{sat}(\boldsymbol{u})$ by the deadzone nonlinearity according to (8) and with $\boldsymbol{u}(t)$ given by (7) the transformed state-space model

$$
\begin{aligned}
\dot{\boldsymbol{x}}_{\mathrm{a}}(t)= & \overline{\boldsymbol{A}}_{\mathrm{a}} \boldsymbol{x}_{\mathrm{a}}(t)+\boldsymbol{B}_{\mathrm{a}} \phi\left(\boldsymbol{K} \boldsymbol{x}_{\mathrm{a}}(t)-\boldsymbol{K}_{\mathrm{P}} \boldsymbol{e}(t)-\boldsymbol{K}_{\mathrm{P}} \boldsymbol{w}(t)\right) \\
& +\overline{\boldsymbol{D}}_{\mathrm{a}} \boldsymbol{w}(t)+\overline{\boldsymbol{F}}_{\mathrm{a}} \boldsymbol{e}(t)+\boldsymbol{E}_{\mathrm{a}} \boldsymbol{d}(t), \quad \boldsymbol{x}_{\mathrm{a}}(0)=\boldsymbol{x}_{\mathrm{a} 0}
\end{aligned}
$$

results with

$$
\begin{aligned}
& \overline{\boldsymbol{A}}_{\mathrm{a}}=\left(\begin{array}{cc}
\boldsymbol{A}+\boldsymbol{B} \boldsymbol{K}_{\mathrm{P}} & \boldsymbol{B} \boldsymbol{K}_{\mathrm{I}} \\
\boldsymbol{I} & \boldsymbol{O}
\end{array}\right) \\
& \overline{\boldsymbol{D}}_{\mathrm{a}}=\overline{\boldsymbol{F}}_{\mathrm{a}}=\left(\begin{array}{c}
-\boldsymbol{B} \boldsymbol{K}_{\mathrm{P}} \\
-\boldsymbol{I}
\end{array}\right) .
\end{aligned}
$$

This model is used in the following stability analysis. Moreover, it is assumed that $\boldsymbol{K}_{\mathrm{P}}$ and $\boldsymbol{K}_{\mathrm{I}}$ are designed such that the matrix $\overline{\boldsymbol{A}}_{\mathrm{a}}$ is Hurwitz.

\section{Stability}

For the sake of simplicity, in the following, the signals $\boldsymbol{w}(t)$ and $\boldsymbol{d}(t)$ are considered to be zero.

Theorem 1: Suppose $\boldsymbol{w}(t)=\boldsymbol{d}(t)=\mathbf{0}, \forall t$. If there exist a symmetric positive definite matrix $\boldsymbol{W} \in \mathbb{R}^{n \times n}$, a positive definite diagonal matrix $\boldsymbol{S} \in \mathbb{R}^{m \times m}$, a matrix $\boldsymbol{Z} \in \mathbb{R}^{m \times n}$, a positive scalar $\eta$ and two a priori fixed positive parameters $\tau_{1}, \tau_{2}$ satisfying

$$
\begin{gathered}
{\left[\begin{array}{ccc}
\boldsymbol{W} \overline{\boldsymbol{A}}_{\mathrm{a}}^{\mathrm{T}}+\overline{\boldsymbol{A}}_{\mathrm{a}} \boldsymbol{W}+\tau_{1} \boldsymbol{W} & \boldsymbol{B}_{\mathrm{a}} \boldsymbol{S}-\boldsymbol{W} \boldsymbol{K}^{\mathrm{T}}-\boldsymbol{Z}^{\mathrm{T}} & \overline{\boldsymbol{F}}_{\mathrm{a}} \\
\star & -2 \boldsymbol{S} & -\boldsymbol{K}_{\mathrm{P}} \\
\star & \tau_{2} \boldsymbol{R}
\end{array}\right]<0} \\
{\left[\begin{array}{cc}
\boldsymbol{W} & \tau_{1} \delta+\tau_{2} \eta<0 \\
\star & \eta u_{0 i}^{2}
\end{array}\right] \geq 0, i \in 1, \ldots, m}
\end{gathered}
$$

then for any $\boldsymbol{e} \in \mathcal{E}=\left\{\boldsymbol{e}: \boldsymbol{e}^{\mathrm{T}} \boldsymbol{R} \boldsymbol{e} \leq \delta^{-1}\right\}$ and $\boldsymbol{x}_{\mathrm{a}}(0) \in \mathcal{R}_{\mathcal{E}}$ with

$$
\mathcal{R}_{\mathcal{E}}=\left\{\boldsymbol{x}_{\mathrm{a}}: \boldsymbol{x}_{\mathrm{a}}^{\mathrm{T}} \boldsymbol{P} \boldsymbol{x}_{\mathrm{a}} \leq \eta^{-1}\right\}
$$

and $\boldsymbol{P}=\boldsymbol{W}^{-1}$, the trajectories of system (9) do not leave the ellipsoid $\mathcal{R}_{\mathcal{E}}$.

Proof: The proof follows the procedure introduced in [15]. Consider the quadratic function

$$
V\left(\boldsymbol{x}_{\mathrm{a}}\right)=\boldsymbol{x}_{\mathrm{a}}^{\mathrm{T}} \boldsymbol{P} \boldsymbol{x}_{\mathrm{a}} .
$$

It has to be proven that $\dot{V}\left(\boldsymbol{x}_{\mathrm{a}}\right)<0$ for any $\boldsymbol{x}_{\mathrm{a}} \notin \operatorname{int} \mathcal{R}_{\mathcal{E}}$ with int $\mathcal{R}_{\mathcal{E}}=\left\{\boldsymbol{x}_{\mathrm{a}}: \boldsymbol{x}_{\mathrm{a}}^{\mathrm{T}} \boldsymbol{P} \boldsymbol{x}_{\mathrm{a}}<\eta^{-1}\right\}$ and any $\boldsymbol{e} \in \mathcal{E}$. According to the $\mathrm{S}$-procedure, the condition

$$
\dot{V}\left(\boldsymbol{x}_{\mathrm{a}}\right)+\tau_{1}\left(\boldsymbol{x}_{\mathrm{a}}^{\mathrm{T}} \boldsymbol{P} \boldsymbol{x}_{\mathrm{a}}-\eta^{-1}\right)+\tau_{2}\left(\delta^{-1}-\boldsymbol{e}^{\mathrm{T}} \boldsymbol{R} \boldsymbol{e}\right)<0
$$

has to be verified which can be split into

$$
\begin{array}{r}
\dot{V}\left(\boldsymbol{x}_{\mathrm{a}}\right)+\tau_{1} \boldsymbol{x}_{\mathrm{a}}^{\mathrm{T}} \boldsymbol{P} \boldsymbol{x}_{\mathrm{a}}-\tau_{2} \boldsymbol{e}^{\mathrm{T}} \boldsymbol{R} \boldsymbol{e} \\
-\tau_{1} \eta^{-1}+\tau_{2} \delta^{-1}<0
\end{array}
$$

the latter of which leads to the second condition (11) of the theorem.

By choosing

$$
\begin{aligned}
\boldsymbol{v} & =\boldsymbol{K} \boldsymbol{x}_{\mathrm{a}}+\boldsymbol{K}_{\mathrm{P}} \boldsymbol{e} \\
\boldsymbol{z} & =\boldsymbol{K} \boldsymbol{x}_{\mathrm{a}}+\boldsymbol{K}_{\mathrm{P}} \boldsymbol{e}+\boldsymbol{G} \boldsymbol{x}_{\mathrm{a}},
\end{aligned}
$$

the nonlinearity $\phi(\boldsymbol{u})$ satisfies the sector condition

$$
\phi(\boldsymbol{u})^{\mathrm{T}} \boldsymbol{T}\left(\phi(\boldsymbol{u})+\boldsymbol{u}+\boldsymbol{G} \boldsymbol{x}_{\mathrm{a}}\right) \leq 0
$$

with $\boldsymbol{u}=\boldsymbol{K} \boldsymbol{x}_{\mathrm{a}}-\boldsymbol{K}_{\mathrm{P}} \boldsymbol{e}$ in the set

$$
\mathcal{R}_{\mathcal{S}}(\|\boldsymbol{G}\|)=\left\{\boldsymbol{x}:\left|\boldsymbol{G}_{i} \boldsymbol{x}\right| \leq u_{0 i}, \quad \forall i=1,2, \ldots m\right\}
$$

according to Lemma 1. As the LMI (12) ensures that the ellipsoid $\mathcal{R}_{\mathcal{E}}$ is included in the set $\mathcal{R}_{\mathcal{S}}(\|G\|)$ the following inequalities hold:

$$
\begin{aligned}
& \dot{V}\left(\boldsymbol{x}_{\mathrm{a}}\right)+\tau_{1} \boldsymbol{x}_{\mathrm{a}}^{\mathrm{T}} \boldsymbol{P} \boldsymbol{x}_{\mathrm{a}}-\tau_{2} \boldsymbol{e}^{\mathrm{T}} \boldsymbol{R} \boldsymbol{e} \\
& \leq \dot{V}\left(\boldsymbol{x}_{\mathrm{a}}\right)+\tau_{1} \boldsymbol{x}_{\mathrm{a}}^{\mathrm{T}} \boldsymbol{P} \boldsymbol{x}_{\mathrm{a}}-\tau_{2} \boldsymbol{e}^{\mathrm{T}} \boldsymbol{R} \boldsymbol{e} \\
& \quad-2 \phi(\boldsymbol{u})^{\mathrm{T}} \boldsymbol{T}\left(\phi(\boldsymbol{u})+\boldsymbol{u}+\boldsymbol{G} \boldsymbol{x}_{\mathrm{a}}\right)<0 .
\end{aligned}
$$

By using the system equation (9) and introducing the change of variables $\boldsymbol{W}=\boldsymbol{P}^{-1}, \boldsymbol{S}=\boldsymbol{T}^{-1}$ and $\boldsymbol{Z}=\boldsymbol{G} \boldsymbol{W}$, finally inequality (10) is obtained. 
Depending on the objective, the result can be used for both

- determining the maximum stability region $\mathcal{R}_{\mathcal{E}}$ for a fixed event threshold $\delta^{-1}$ and

- determining the maximum event threshold $\delta^{-1}$ for which the inequalities (10)-(12) are feasible.

Note that the size of the ellipsoid $\mathcal{R}_{\mathcal{E}}$ might severely depend on the selection of the parameters $\tau_{1}, \tau_{2}$. Therefore, in order to obtain the maximum region of stability, a grid using different pairs $\tau_{1}, \tau_{2}$ needs to be defined based on which an optimization (see Sec. V) has to be carried out for every grid point.

\section{E. Minimum inter-event time}

The minimum time interval between two consecutive events (minimum inter-event time) is given by

$$
T_{\min }=\min _{k}\left\{t_{k+1}-t_{k}\right\}, \quad k=0,1,2, \ldots .
$$

The next theorem shows that there exists a lower bound on the minimum inter-event time and, hence, Zeno behavior can be excluded.

Theorem 2: Assume that the event-triggered PI-control loop (9) with $\boldsymbol{w}(t)=\boldsymbol{d}(t)=\mathbf{0}$ satisfies the inequalities (10)(12), then for $\boldsymbol{x}_{\mathrm{a}}(0) \in \mathcal{R}_{\mathcal{E}}$ the minimum inter-event time $T_{\min }$ is lower bounded by

$$
T_{\min } \geq \bar{T}=\arg \min _{t}\left\{\tilde{e}(t)=\sqrt{\frac{1}{\delta\|\boldsymbol{R}\|}}\right\}
$$

with

$$
\begin{aligned}
\tilde{e}(t)= & \max _{t}\left\|\mathrm{e}^{\boldsymbol{A} t}-\boldsymbol{I}_{n}\right\| x_{\max } \\
& +\int_{0}^{t}\left\|\mathrm{e}^{\boldsymbol{A}(t-\tau)}\right\| \mathrm{d} \tau\|\boldsymbol{B}\| u_{0}
\end{aligned}
$$

and

$$
x_{\max }=\max _{\boldsymbol{x} \in \mathcal{R}_{\mathcal{E}}}\|\boldsymbol{x}\| .
$$

Proof: The behavior of the plant is given by

$$
\dot{\boldsymbol{x}}(t)=\boldsymbol{A} \boldsymbol{x}(t)+\boldsymbol{B} \tilde{\boldsymbol{u}}(t)+\boldsymbol{E} \boldsymbol{d}(t), \quad \boldsymbol{x}(0)=\boldsymbol{x}_{0}
$$

(see (1)) with $\tilde{\boldsymbol{u}}(t)$ a saturation nonlinearity according to (2). For $\boldsymbol{d}(t)=\mathbf{0}$, the solution of this differential equation is given by

$$
\boldsymbol{x}(t)=\mathrm{e}^{\boldsymbol{A} t} \boldsymbol{x}_{0}+\int_{0}^{t} \mathrm{e}^{\boldsymbol{A}(t-\tau)} \boldsymbol{B} \tilde{\boldsymbol{u}}(\tau) \mathrm{d} \tau .
$$

The norm of the state error

$$
\|\boldsymbol{e}(t)\|=\left\|\boldsymbol{x}(t)-\boldsymbol{x}\left(t_{k}\right)\right\|
$$

introduced by considering the over-approximation

$$
\boldsymbol{e}^{\mathrm{T}} \boldsymbol{R} \boldsymbol{e} \leq\|\boldsymbol{e}\|^{2}\|\boldsymbol{R}\|
$$

can be upper bounded by

$$
\begin{aligned}
\|\boldsymbol{e}(t)\| & =\left\|\left(\mathrm{e}^{\boldsymbol{A} t}-\boldsymbol{I}_{n}\right) \boldsymbol{x}_{0}+\int_{0}^{t} \mathrm{e}^{\boldsymbol{A}(t-\tau)} \boldsymbol{B} \tilde{\boldsymbol{u}}(\tau) \mathrm{d} \tau\right\| \\
& \leq \tilde{e}(t)
\end{aligned}
$$

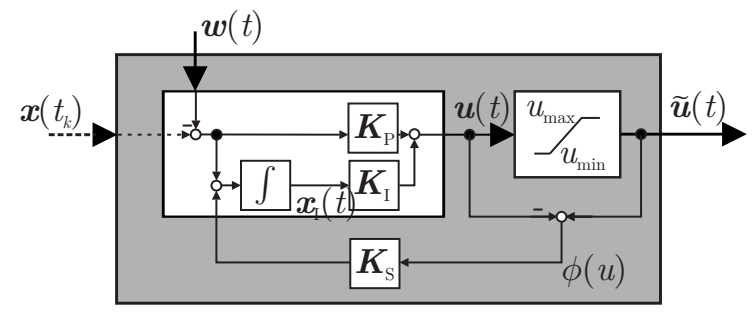

Fig. 3. Static anti-windup control.

$\left(t_{k}=0\right)$ with $\tilde{e}(t)$ given by (15). As an event is generated whenever the equation $\boldsymbol{e}^{\mathrm{T}} \boldsymbol{R} \boldsymbol{e}=\delta^{-1}$ holds, $\tilde{e}(t)$ can be used to derive a lower bound on the minimum inter-event time by means of relation (14).

\section{ANTI-WINDUP CONTROL}

\section{A. Modification of the components}

In order to compensate the potential performance degradation due to saturating actuators, anti-windup techniques have been developed (see [4], [20]). This section applies a static anti-windup mechanism to the event-triggered PIcontrol loop and investigates how the event-triggered control is affected by this extension.

The anti-windup mechanism is illustrated in Fig. 3. It uses the difference between the saturated input $\tilde{\boldsymbol{u}}(t)$ and the input signal $\boldsymbol{u}(t)$ produced by the controller, i.e., $\phi(\boldsymbol{u})=\operatorname{sat}(\boldsymbol{u})-$ $\boldsymbol{u}$ (see (8)), to affect the integrator state $\boldsymbol{x}_{\mathrm{I}}(t)$ through the feedback matrix $\boldsymbol{K}_{\mathrm{S}} \in \mathbb{R}^{n \times m}$. Hence, only the first equation of controller (4), (5) has to be modified:

$$
\begin{aligned}
\dot{\boldsymbol{x}}_{\mathrm{I}}(t) & =\boldsymbol{x}(t)-\boldsymbol{e}(t)-\boldsymbol{w}(t)+\boldsymbol{K}_{\mathrm{S}} \phi(\boldsymbol{u}(t)), \boldsymbol{x}_{\mathrm{I}}(0)=\boldsymbol{x}_{\mathrm{I} 0}(17) \\
\boldsymbol{u}(t) & =\boldsymbol{K}_{\mathrm{I}} \boldsymbol{x}_{\mathrm{I}}(t)+\boldsymbol{K}_{\mathrm{P}}(\boldsymbol{x}(t)-\boldsymbol{e}(t)-\boldsymbol{w}(t))
\end{aligned}
$$

\section{B. Model of the extended control loop}

With plant (1) and controller (17), (18) and by using the deadzone nonlinearity according to (8), the event-triggered PI-control loop with anti-windup compensation is described by the state-space model

$$
\begin{aligned}
\dot{\boldsymbol{x}}_{\mathrm{a}}(t)= & \overline{\boldsymbol{A}}_{\mathrm{a}} \boldsymbol{x}_{\mathrm{a}}(t)+\overline{\boldsymbol{B}}_{\mathrm{a}} \phi\left(\boldsymbol{K} \boldsymbol{x}_{\mathrm{a}}(t)-\boldsymbol{K}_{\mathrm{P}} \boldsymbol{e}(t)-\boldsymbol{K}_{\mathrm{P}} \boldsymbol{w}(t)\right) \\
& +\overline{\boldsymbol{D}}_{\mathrm{a}} \boldsymbol{w}(t)+\overline{\boldsymbol{F}}_{\mathrm{a}} \boldsymbol{e}(t)+\boldsymbol{E}_{\mathrm{a}} \boldsymbol{d}(t), \boldsymbol{x}_{\mathrm{a}}(0)=\boldsymbol{x}_{\mathrm{a} 0} .
\end{aligned}
$$

The only difference to model (9) is given by replacing $\boldsymbol{B}_{\mathrm{a}}$ by $\bar{B}_{\text {a }}$ with

$$
\overline{\boldsymbol{B}}_{\mathrm{a}}=\left(\begin{array}{c}
\boldsymbol{B} \\
\boldsymbol{K}_{\mathrm{S}}
\end{array}\right) .
$$

\section{Stability}

As the anti-windup extension only replaces the matrix $\boldsymbol{B}_{\text {a }}$ by $\bar{B}_{\mathrm{a}}$ the result stated in Theorem 1 can be simply adopted to the anti-windup scenario.

Corollary 1: Suppose $\boldsymbol{w}(t)=\boldsymbol{d}(t)=\mathbf{0}, \forall t$. If there exist a symmetric positive definite matrix $\boldsymbol{W} \in \mathbb{R}^{n \times n}$, a positive definite diagonal matrix $\boldsymbol{S} \in \mathbb{R}^{m \times m}$, a matrix $\boldsymbol{Z} \in \mathbb{R}^{m \times n}$, a positive scalar $\eta$ and two a priori fixed positive parameters $\tau_{1}, \tau_{2}$ satisfying

$$
\left[\begin{array}{ccc}
\boldsymbol{W} \overline{\boldsymbol{A}}_{\mathrm{a}}^{\mathrm{T}}+\overline{\boldsymbol{A}}_{\mathrm{a}} \boldsymbol{W}+\tau_{1} \boldsymbol{W} & \overline{\boldsymbol{B}}_{\mathrm{a}} \boldsymbol{S}-\boldsymbol{W} \boldsymbol{K}^{\mathrm{T}}-\boldsymbol{Z}^{\mathrm{T}} & \overline{\boldsymbol{F}}_{\mathrm{a}} \\
\star & -2 \boldsymbol{S} & -\boldsymbol{K}_{\mathrm{P}} \\
\star & \star & -\tau_{2} \boldsymbol{R}
\end{array}\right]<0
$$




$$
\begin{gathered}
-\tau_{1} \delta+\tau_{2} \eta<0 \\
{\left[\begin{array}{cc}
\boldsymbol{W} & \boldsymbol{Z}_{i}^{\mathrm{T}} \\
\star & \eta u_{0 i}^{2}
\end{array}\right] \geq 0, i \in 1, \ldots, m}
\end{gathered}
$$

then for any $\boldsymbol{e} \in \mathcal{E}=\left\{\boldsymbol{e}: \boldsymbol{e}^{\mathrm{T}} \boldsymbol{R} \boldsymbol{e} \leq \delta^{-1}\right\}$ and $\boldsymbol{x}_{\mathrm{a}}(0) \in \mathcal{R}_{\mathcal{E}}$ with $\mathcal{R}_{\mathcal{E}}$ given by (13) and $\boldsymbol{P}=\boldsymbol{W}^{-1}$, the trajectories of system (19) do not leave the ellipsoid $\mathcal{R}_{\mathcal{E}}$.

Note that $\boldsymbol{K}_{\mathrm{S}}$ now introduces additional degrees of freedom by affecting $\overline{\boldsymbol{B}}_{\text {a }}$ according to (20) which can be used to improve the behavior of the event-triggered control loop, e.g., by increasing the size of the region of stability as shown later in the simulation.

Moreover, the anti-windup scheme does not directly affect the bound on the inter-event time as the bound is derived by means of the bound $\boldsymbol{u}_{0}$ on the control input $\tilde{\boldsymbol{u}}$. Hence, Theorem 2 holds in the anti-windup case as well.

Corollary 2: Assume that the event-triggered control loop (19) with $\boldsymbol{w}(t)=\boldsymbol{d}(t)=\mathbf{0}$ satisfies the inequalities (21)-(23), then for $\boldsymbol{x}_{\mathrm{a}}(0) \in \mathcal{R}_{\mathcal{E}}$ the minimum inter-event time $T_{\min }$ is lower bounded by $\bar{T}$ given by (14).

However, as the extension might increase the ellipsoid $\mathcal{R}_{\mathcal{E}}$ it indirectly affects the bound through a potentially larger parameter $x_{\max }$ (see (16)).

\section{NUMERICAL EXAMPLE}

\section{A. Plant description}

In [9] is has been shown by simulation that actuator saturation might significantly affect the behavior of the eventtriggered PI-control loop which, moreover, strongly depends on the selection of the event threshold. This section considers the same scalar plant model which is given by

$$
\dot{x}(t)=0.1 x(t)+\tilde{u}(t), \quad x(0)=0 .
$$

The plant input is affected by the actuator saturation

$$
\tilde{u}(t)=\operatorname{sat}(u(t))= \begin{cases}0.4, & \text { for } u(t)>0.4 \\ u(t), & \text { for }-0.4 \leq u(t) \leq 0.4 \\ -0.4, & \text { for } u(t)<-0.4\end{cases}
$$

and the PI controller is given by

$$
\begin{aligned}
\dot{x}_{\mathrm{I}}(t) & =x(t), \quad x_{\mathrm{I}}(0)=x_{\mathrm{I} 0} \\
u(t) & =-x_{\mathrm{I}}(t)-1.6 x(t)
\end{aligned}
$$

with $w(t)=0$. An event is generated whenever $(e(t))^{2}=$ $\delta^{-1}$ holds $(r=1)$ which, by setting $\delta^{-1}=\bar{e}^{2}$, corresponds to the original deadband sampling rule [14], i.e.,

$$
\left|x(t)-x\left(t_{k}\right)\right|=\bar{e}
$$

with $\bar{e}$ the event threshold.

\section{B. Consequences of actuator saturation}

Figure 4 shows the stability regions for different values of the event threshold $\bar{e}$. The regions have been obtained by solving the optimization problem

\section{$\max \operatorname{trace}(\boldsymbol{W})$}

subject to inequalities (10) - (12)

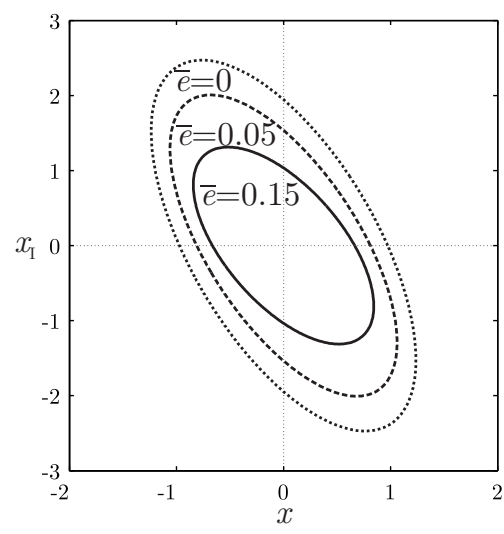

Fig. 4. Stability regions for different event thresholds; $\bar{e}=0$ indicates the region for the corresponding continuous-time PI-control loop.
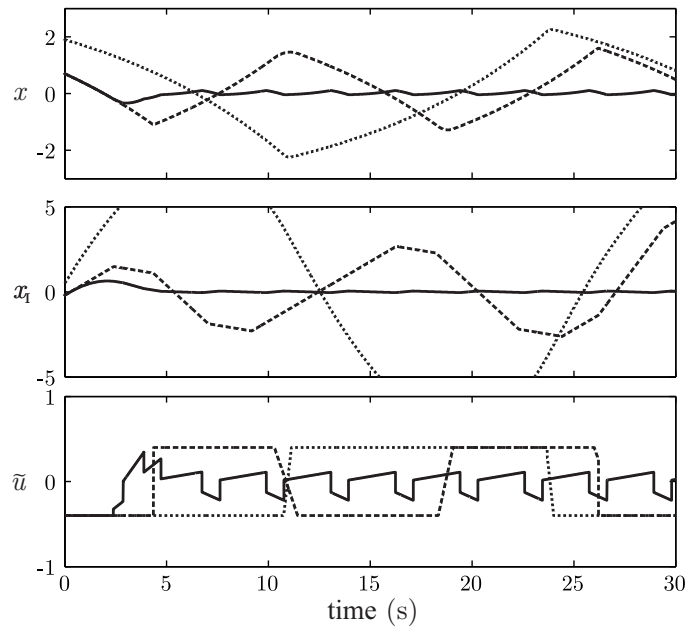

Fig. 5. Behavior of the event-triggered PI-control loop for: 1. $\bar{e}=0.15$, $\boldsymbol{x}_{0} \in \mathcal{R}_{\mathcal{E}}(0.15)$ (solid line), 2. $\bar{e}=0.9, \boldsymbol{x}_{0} \in \mathcal{R}_{\mathcal{E}}(0.15)$ (dashed line), 3. $\bar{e}=0.15, \boldsymbol{x}_{0} \notin \mathcal{R}_{\mathcal{E}}(0.15)$ (dotted line).

with the YALMIP toolbox [11]. The figure indicates that the size of the region decreases by increasing the event threshold, where $\bar{e}=0.15$ denotes the maximum threshold for which inequalities (10)-(12) yield a feasible solution. The region obtained for $\bar{e}=0.15$ is denoted in the following by $\mathcal{R}_{\mathcal{E}}(0.15)$.

The behavior of the event-triggered PI-control loop is depicted in Fig. 5. Here, the upper plot shows the plant state $x(t)$. The integrator state $x_{\mathrm{I}}(t)$ is depicted in the middle plot.

Three different scenarios are considered. The behavior using $\bar{e}=0.15$ with $\boldsymbol{x}_{0} \in \mathcal{R}_{\mathcal{E}}(0.15)$ is drawn by the solid lines. As expected the behavior remains stable. The dashed lines indicate the behavior obtained by starting from the same initial condition but with an increased event threshold $(\bar{e}=$ 0.9). The behavior becomes unstable. The same holds by choosing an admissible event threshold $\bar{e}=0.15$ but considering an initial state $\boldsymbol{x}_{0} \notin \mathcal{R}_{\mathcal{E}}(0.15)\left(\boldsymbol{x}_{0}=\left(\begin{array}{ll}1.9 & 0.5\end{array}\right)^{\mathrm{T}}\right)$ depicted by the dotted lines. In the latter two cases the instability results from the fact that the input signal $\tilde{u}(t)$ is kept almost always in its saturation bounds (see lower plot of the figure) which is caused by a large integrator state $x_{\mathrm{I}}$. 


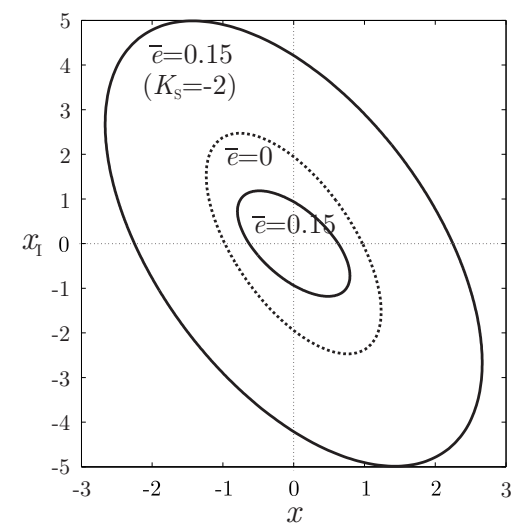

Fig. 6. Increase of the stability region due to anti-windup compensation.

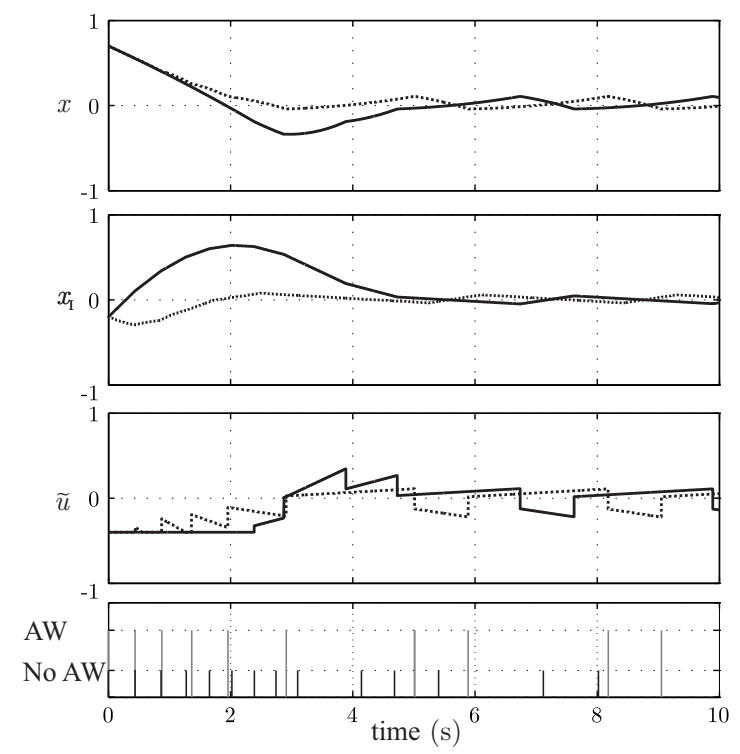

Fig. 7. Behavior the event-triggered PI-control loop with (dotted lines) and without (solid lines) anti-windup compensation.

\section{Anti-windup control}

Figure 6 shows how the anti-windup extension affects the region of stability. The region is significantly increased and even includes the region obtained for continuous-time PI control without compensation which is depicted for

$$
K_{\mathrm{S}}=-2 .
$$

Moreover, Fig. 7 compares the resulting trajectories obtained without and with anti-windup control $\left(K_{\mathrm{S}}=-2\right)$ for $\boldsymbol{x}_{0} \in$ $\mathcal{R}_{\mathcal{E}}(0.15)$ and $\bar{e}=0.15$. It is shown that the overshoot with anti-windup control is much smaller compared to the scheme without compensation (upper plot) as the integrator state is much smaller (second plot). This leads to a significant shorter period in which the actuator is saturated which can be seen at the beginning of the simulation (third plot). The lower plot of Fig. 7 shows the event times. The anti-windup control (AW) decreases the overall number of events ( 9 instead of 13 events in the time interval considered) primarily by reducing the communication during the transient behavior.

\section{CONCLUSIONS}

In this paper, the influence of actuator saturation on event-triggered PI control has been investigated. As a main result LMI conditions have been derived which allow to determine regions of stability for the event-triggered closedloop system. Simulations have shown that by incorporating a static anti-windup mechanism the region of stability and the overall performance of the event-triggered PI control can be significantly improved.

Future work will include alternative methods for deriving the stability regions and the design of event-triggered antiwindup mechanisms.

\section{REFERENCES}

[1] A. Anta and P. Tabuada. To sample or not to sample: Self-triggered control for nonlinear systems. IEEE Transactions on Automatic Control, 55(9):2030-2042, 2010.

[2] K.-E. Årzén. A simple event-based PID controller. In Proceedings of IFAC World Congress, pages 423-428, Beijing, 1999.

[3] K. J. Åström and B. Bernhardsson. Comparison of Riemann and Lebesgue sampling for first order stochastic systems. In Proceedings of IEEE Conference on Decision and Control, Vol. 2, pages 20112016, Las Vegas, 2002.

[4] K. J. Åström and T. Hägglund. PID Controllers: Theory, Design, and Tuning. ISA, 1995.

[5] A. Bemporad, W. P. M. H. Heemels, and M. Johansson. Networked Control Systems. Lecture Notes in Control and Information Sciences. Springer Verlag, 2010

[6] A. Cervin and T. Henningsson. Scheduling of event-triggered controllers on a shared network. In Proceedings of IEEE Conference on Decision and Control, pages 3601-3606, Cancun, 2008.

[7] M. C. F. Donkers and W. P. M. H. Heemels. Output-based eventtriggered control with guaranteed $\mathcal{L}_{\infty}$-gain and improved eventtriggering. In IEEE Conference on Decision and Control, Atlanta, 2010.

[8] W. P. M. H. Heemels, J. Sandee, and P. van den Bosch. Analysis of event-driven controllers for linear systems. Intern. J. of Control, 81(4):571-590, 2008.

[9] D. Lehmann and K. H. Johansson. Event-triggered PI control subject to actuator saturation. In Proceedings of IFAC Conference on Advances in PID Control, Brescia, 2012.

[10] D. Lehmann and J. Lunze. Extension and experimental evaluation of an event-based state-feedback approach. Control Engineering Practice, 19(2):101-112, 2011.

[11] J. Löfberg. YALMIP : A toolbox for modeling and optimization in MATLAB. In Proceedings of the CACSD Conference, Taipei, 2004.

[12] J. Lunze and D. Lehmann. A state-feedback approach to event-based control. Automatica, 46(1):211-215, 2010.

[13] G. N. Nair, F. Fagnani, S. Zampieri, and R. J. Evans. Feedback control under data rate constraints: an overview. Proceeding of the IEEE, 95(1):108-137, 2007.

[14] P. G. Otanez, J. G. Moyne, and D. M. Tilbury. Using deadbands to reduce communication in networked control systems. In Proceedings of American Control Conference, pages 3015-3020, Anchorage, 2002.

[15] S. Tarbouriech, G. Garcia, J. M. Gomes da Silva Jr., and I. Queinnec. Stability and Stabilization of Linear Systems with Saturating Actuators. Springer, 2011.

[16] U. Tiberi, J. Araújo, and K. H. Johansson. On event-based PI control of first-order processes. In Proceedings of IFAC Conference on Advances in PID Control, Brescia, 2012.

[17] Y. Tipsuwan and M. Y. Chow. Control methodologies in networked control systems. Control Engineering Practice, 11:1099-1111, 2003.

[18] V. Vasyutynskyy and K. Kabitzsch. Implementation of PID controller with send-on-delta sampling. In Proceedings of International Control Conference, Glasgow, 2006.

[19] X. Wang and M. D. Lemmon. Self-triggered feedback control systems with finite-gain $\mathcal{L}_{2}$ stability. IEEE Transactions on Automatic Control, 54(3):452-467, 2009.

[20] L. Zaccarian and A. R. Teel. Modern Anti-windup Synthesis: Control Augmentation for Actuator Saturation. Princeton, 2011. 